\title{
Editorial
}

\section{Modern applications of copula and financial modeling}

Copula modeling has been attracting substantial interest during the last several decades and is becoming a regular tool for modeling statistical dependence among various applications including insurance (joint life policies and annuities), finance (extreme co-movements of stock prices and exchange rates as well as credit derivative pricing), and engineering (related failures in complex systems). The main advantage of the copula approach is its ability to estimate the entire joint distribution using marginal distributions and the association parameter, making it possible for researchers to go beyond correlation and detect complex non-linear dependence patterns.

The papers presented in this issue focus on current applications of copula analysis in risk management and finance, modeling of asymmetrical and directional dependence, and new approaches to copula model selection.

The paper by I.K. Andrievskaya and H.I. Penikas deals with modeling the Russian banking system's capital adequacy under the Basel II regulatory requirements and analyzes their pro-cyclicality effect. Copulas are used to model the joint banking risk distribution which shows the downward change in the risks' interconnection associated with the increasing stability of the banking system.

M. Jadin, P. Persons, and J. Woychik describe a case study on the dependence of index prices for Asian markets. Bivariate copulas are used to model the pair-wise association between national market indices. Empirical analysis based on Kendall's concordance coefficient is used for copula model selection. A similar study involving several European markets is performed by V. Gordeev, A. Kniazev, and A. Shemyakin. The focus of their paper is on the comparison of the results of full parametric and semi-parametric estimation, and also on several empirical criteria for model selection.

D. Uhm, J-M. Kim, and Y-S. Jung examine asymmetry in financial data applying copulas to define directional dependence. Together with tail dependence, the directional dependence describes the behavior of currency exchange rates in Korea and Japan versus the US dollar. In an innovative methodological development, E.A. Sungur and J.M. Orth introduce a new way of looking at directional dependence by using a direction parameter expressed as an angle.

The paper from T.N. Matcharashvili, N.I. Jibladze, T.G. Iluridze, T.T. Matcharashvili and A.L. Topchishvili is devoted to the investigation of statistical, distributional and dynamic features of the exchange rate variation of the Georgian currency to the US Dollar within the last 15 years. The probability distribution used to fit the observed exchange rate data to various hypothesized statistical models and statistical quality characteristics help to identify the best distribution for a given scenario. Scaling and dynamic features of the exchange rate's variation are defined using linear and nonlinear methods of time series analysis.

These papers present many modern developments in copula analysis and financial modeling which we hope readers will find useful in their own applications and research.

Dr. Arkady Shemyakin

Guest Editor for MASA \#4, Volume 7, 2012 Professor, University of St. Thomas St. Paul, MN, USA

Dr. Stan Lipovetsky MASA Co-Editor-in-Chief, Minneapolis, MN, USA 